\title{
Profile of Tinea Corporis and Tinea Cruris in Dermatovenereology Clinic of Tertiery Hospital: A Retrospective Study
}

\author{
Selvia Yulianti Dwi Ratih Sanggarwati ${ }^{1}$, Manik Retno Wahyunitisari ${ }^{2}$, Linda Astari $^{3}$, \\ Evy Ervianti ${ }^{3}$ \\ ${ }^{1}$ Undergraduate of Medicine Program, Faculty of Medicine Universitas Airlangga \\ ${ }^{2}$ Departement of Microbiology, Faculty of Medicine, Universitas Airlangga, Dr. Soetomo General \\ Academic Teaching Hospital, Surabaya, Indonesia \\ ${ }^{3}$ Departement of Dermatovenereology, Faculty of Medicine, Universitas Airlangga, Dr. Soetomo \\ General Academic Teaching Hospital, Surabaya, Indonesia
}

\begin{abstract}
Background: Tinea corporis and cruris are dermatophytes that infect the skin, and they are caused by Trichophyton sp., Microsporum sp., and Epidermophyton sp. Dermatophyte fungal-infect keratin-containing skin classified by the body's location, such as tinea corporis (skin besides haired area, body, hands, or feet) and tinea cruris (groin \& perineal region). Purpose: To evaluate the clinical profiles and treatments of tinea corporis and cruris at Dermatology and Venereology (DV) outpatient clinic Dr. Soetomo General Academic Hospital in 2018. Methods: A retrospective-descriptive study based on medical records of DV outpatient clinic Dr. Soetomo General Academic Hospital, Surabaya. Data collected from August to December 2019. Tinea corporis and cruris profiles evaluation based on age, sex, occupation, precipitating factors, complaints, illness duration, family history, lesion's location and description, and therapy. Result: A total of 164 patients (35 tinea corporis, 76 tinea cruris, and 53 tinea corporis and cruris), predominantly female, age 46-55 years, came treatment less-than-a-month cases, excessive sweating, itching, tinea corporis lesion's location on the face and body, tinea cruris in groin, tinea corporis and cruris on body and groin, erythematous macules, firm borders, active margins, scales, central healing, positive $\mathrm{KOH} 10$ $20 \%$ examination, miconazole cream therapy $2 \%$, griseofulvin therapy, griseofulvin oral and ketoconazole cream $2 \%$ combination therapy. Discussion: Tinea corporis and cruris are common in females as their history and symptoms supported by the characteristics of laboratory examination, the presence of hyphae in potassium hydroxide (KOH) $10-20 \%$. Most patients were prescribed with oral griseofulvin, especially in large lesions cases.
\end{abstract}

Keywords: tinea corporis, tinea cruris, potassium hydroxide $10-20 \%$, retrospective descriptive research, Dr. Soetomo General Academic Hospital.

Correspondence: Evy Ervianti, Department/SMF of Department of Dermatology and Venereology, Faculty of Medicine, Universitas Airlangga/Dr. Soetomo General Academic Hospital, Jl. Mayjen Prof. Dr. Moestopo No. 6-8, Airlangga, Gubeng, Surabaya, East Java 60286, Phone: 081332681513, E-mail : evy_if@yahoo.co.id.

\section{BACKGROUND}

Tinea corporis and cruris are dermatophyte infections of the skin. They are caused by fungi, Trichophyton sp., Microsporum sp., and Epidermophyton sp. ${ }^{1}$ Dermatophyte fungal infections can be classified in regards to their location, such as tinea corporis (skin area other than hairy areas, body, hands, or feet) and tinea cruris (groin and perineal area). ${ }^{2}$ Dermatophytosis infection has spread worldwide. The prevalence of this disease is $20-25 \%$ with the most common cause is dermatophyte fungi, while the most common in Asia is tinea corporis infection with a prevalence of $35.40 \%{ }^{3}$ In 2008-2010 Citrashanty mentioned that, at the Mycology Division of Dermatology and Venereology Outpatient Clinic Dr. Soetomo General Academic Hospital Surabaya, $59.5 \%, 56.3 \%$, and $52.9 \%$ of them were dermatophytosis cases. This was then confirmed by the data at the Dr. Soetomo General Academic Hospital Surabaya in 2014-2016, among others, amounted to $47.4 \%, 52.9 \%$, and $46 \%$. This proves that almost half of the cases in the Mycology Division were dermatophytosis cases. ${ }^{4}$

Dermatophytosis transmission spreads in 3 ways, namely anthropophilic, zoophilic, and geophilic. Anthropophilic transmission means human-to-human transmission, zoophilic means animal-to-human transmission, and geophilic means soil-to-human transmission. ${ }^{4,5}$ Tinea corporis and cruris are chronic, and many patients experience failure of therapy. Such failure was not only due to patient non-compliance, but also because of non-upright or often wrong diagnoses. As a result, the patient's quality would decline. Therefore, it is necessary to evaluate the clinical profile 
of the two diseases to decrease their prevalence and to finalise their diagnoses.

\section{METHODS}

This was a retrospective descriptive study based on medical records at the Dermatology and Venereology Outpatient Clinic Dr. Soetomo General Academic Hospital. The data collection was carried out in August-December 2019. The profile analysis of tinea corporis and cruris of outpatients was carried out by completing records of the patient's age, sex, occupation, trigger factors, main complaints, length of illness, family history, location of the lesion, description of the lesion, investigations, and therapy. This study has received ethical clearance from the Health Research Ethics Committee of Dr. Soetomo General Academic Hospital Surabaya No. $1517 / \mathrm{KEPK} / \mathrm{IX} / 2019$. The medical records of tinea corporis and cruris patients were then be grouped based on those criteria and presented in diagrams and tables.

\section{RESULT}

A total of 164 medical records were obtained, consisting of 35 tinea corporis patients, 76 tinea cruris patients, and 53 tinea corporis and cruris tinea patients. Of the 164 patients, most of the patients were females (59.1\%), under the age group of 46-55 years (20.7\%), private employee $(38.4 \%)$, came for therapy when the duration of illness was less than 1 month (29.2\%), no family history $(71.3 \%)$, excessive sweating as the triggering factor $(43.3 \%)$, itchiness as the main complaint $(89.6 \%)$, the location of tinea corporis lesions on the face and body $(22.9 \%)$, tinea cruris in the groin $(55.3 \%)$, and tinea corporis and cruris on the body and groin (26.4\%), erythematous macular lesions, firm boundaries, active margins, scales, central healing (20.7\%), potassium hydroxide $(\mathrm{KOH}) 10-20 \%$ tests positive $(80.4 \%)$, miconazole cream $2 \%$ (7\%) topical therapy, griseofulvin systemic therapy (69\%), and combination therapy griseofulvin tablet and ketoconazole cream $2 \%$ (3.04\%).

There were 76 tinea cruris patients $(46.3 \%), 53$ patients with tinea corporis and cruris $(32.3 \%)$, and 35 tinea corporis patients $(21.4 \%)$. The sex distribution of the patients shows consisting of 67 males $(40.9 \%)$ and 97 females (59.1\%). In terms of sex, there were 29 tinea corporis male patients $(74.3 \%), 46$ tinea cruris female patients $(60.5 \%)$, and 28 tinea corporis and cruris male patients $(52.8 \%)$.

The highest prevalence tinea corporis was under the middle-old age group, which was 56-65 years old, as many as 9 patients $(25.7 \%)$. The youngest tinea corporis patient was 1 year old, and the oldest was 69 years old. The tinea cruris patients indicated that the majority were the youngest-old group, namely 17 patients $(22.4 \%)$. The youngest patient of tinea cruris patients was 5 months and the oldest was 72 years. The sample of tinea corporis and cruris patients showed that the largest age group was the youngest-old and early adult categories, namely 12 patients $(22.6 \%)$. The youngest patient in the tinea corporis and cruris group was 3 years old while the oldest was 117 years.

Most of the patients worked as private employees. There were 14 tinea corporis patients (40\%), 26 tinea cruris patients $(34.2 \%)$, and 23 patients $(43.4 \%)$ with tinea corporis and cruris. Figure 1 shows that most of the patients had excessive sweating, and 10 of them $(28.6 \%)$ had tinea corporis, 34 of them $(44.7 \%)$ had tinea cruris, and 27 of them (50.9\%) had tinea corporis and cruris.

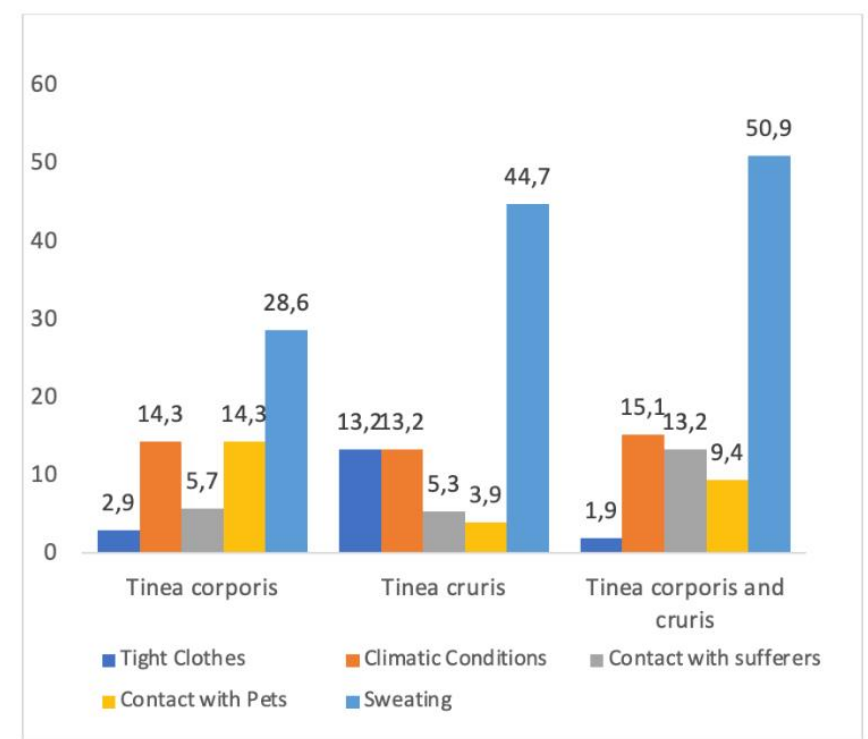

Figure 1. The triggering factors of tinea corporis and cruris. 
Most the chief complaints are itchiness, tinea corporis 31 patients $(88,6 \%)$, tinea cruris 66 patients $(86,8 \%)$, tinea corporis and cruris are 50 patients $(94,3 \%)$. The location of lesion for tinea corporis located in the face and body as observed in 8 patients
(22.9\%). Location of tinea cruris cases located on the groin, as observed in 42 patients $(55.3 \%)$. Location of tinea corporis and cruris were located in the trunk and groin, as observed in 14 patients $(26.4 \%)$.

Table 1. Family history of tinea corporis and cruris

\begin{tabular}{llll}
\hline Family history & Tinea corporis & Tinea cruris & Tinea corporis and cruris \\
\hline Yes & $1(2.9 \%)$ & $1(1.3 \%)$ & $3(5.7 \%)$ \\
No & $24(68.6 \%)$ & $61(80.3 \%)$ & $32(60.4 \%)$ \\
No data & $10(28.6 \%)$ & $14(18.4 \%)$ & $12(22.6 \%)$ \\
\hline
\end{tabular}

Table 2. The laboratory examination of $10-20 \% \mathrm{KOH}$ and SDA culture

\begin{tabular}{llccc}
\hline & Examination & Positive & Negative & No data \\
\hline Tinea corporis & $10-20 \% \mathrm{KOH}$ & $29(82.9 \%)$ & $4(11.4)$ & $2(5.7 \%)$ \\
& SDA culture & - & - & - \\
\hline Tinea cruris & $10-20 \% \mathrm{KOH}$ & $60(78.9 \%)$ & $11(14.5 \%)$ & $5(6.6 \%)$ \\
& SDA culture & - & - & - \\
\hline Tinea corporis and cruris & $10-20 \% \mathrm{KOH}$ & $43(81.1 \%)$ & $7(13.2 \%)$ & $3(5.7 \%)$ \\
& SDA culture & - & - & - \\
\hline
\end{tabular}

$\mathrm{KOH}=$ Potassium hydroxide; SDA = Sabouraud dextrose agar

Table 3. The treatment of tinea corporis

\begin{tabular}{|c|c|}
\hline Treatment & Total $(\%)$ \\
\hline \multicolumn{2}{|l|}{ Systemic treatment } \\
\hline Griseofulvin tablet & $21(60 \%)$ \\
\hline Griseofulvin tablet and cetirizine tablet & $2(5.7 \%)$ \\
\hline Griseofulvin tablet and vitamin B1 tablet & $1(2.9 \%)$ \\
\hline Griseofulvin tablet and loratadine tablet & $1(2.9 \%)$ \\
\hline Griseofulvin tablet and chlorpheniramine maleate tablet & $1(2.9 \%)$ \\
\hline Ketoconazole tablet & $3(8.6 \%)$ \\
\hline Combination treatment & $2(5.7 \%)$ \\
\hline No systemic treatment & $3(8.6 \%)$ \\
\hline No data & $1(2.9 \%)$ \\
\hline \multicolumn{2}{|l|}{ Topical treatment } \\
\hline Ketoconazole $2 \%$ cream & $3(8.6 \%)$ \\
\hline Miconazole $2 \%$ cream & $4(11.4 \%)$ \\
\hline Combination treatment & $2(5.7 \%)$ \\
\hline No topical treatment & $25(71.4 \%)$ \\
\hline No data & $1(2.9 \%)$ \\
\hline \multicolumn{2}{|l|}{ Combination treatment } \\
\hline Griseofulvin tablet and ketoconazole $2 \%$ cream & $3(8.6 \%)$ \\
\hline Griseofulvin tablet and miconazole $2 \%$ cream & $3(8.6 \%)$ \\
\hline No data & $1(2.9 \%)$ \\
\hline No combination treatment & $28(80 \%)$ \\
\hline
\end{tabular}

The most clinical features of tinea corporis lesions were erythematous macules, firm borders, active margins, scales, central healing, as observed in 5 patients $(14.3 \%)$. Tinea cruris lesions were erythematous macules, firm borders, active margins, scales, central healing, as observed in 24 patients $(31.6 \%)$. Tinea corporis and cruris lesions were erythematous macules, firm borders, active margins, scales, central healing, as observed in 5 patients (9.4\%). Duration of illness of tinea corporis and cruris 
patients sought treatment in less than 1 month after the illness started, consisting of 9 tinea corporis patients $(25.7 \%), 26$ tinea cruris patients $(34.2 \%)$, and 13 patients $(24.5 \%)$ with both tinea corporis and cruris. Table 1 shows that most of the patients had no family history of the disease, totalling 24 tinea corporis patients $(68.6 \%), 61$ tinea cruris patients $(80.3 \%), 32$ patients $(60.4 \%)$ with both tinea corporis and cruris. Table 2 shows the $\mathrm{KOH}$ examination of tinea corporis showed 29 positive results $(82.9 \%), 60$ positive results on tinea cruris, and 43 positive results on patients with tinea corporis and tinea cruris $(81.1 \%)$. Table 3 shows that most patients received systemic therapy with griseofulvin, as observed in 21 patients $(60 \%)$.

The most used topical therapy was miconazole $2 \%$ cream, as observed in with 4 patients $(11.4 \%)$ each. 6 patients received combined therapy of griseofulvin

Table 4. The treatment of tinea cruris

\begin{tabular}{|c|c|}
\hline Treatment & Total $(\%)$ \\
\hline \multicolumn{2}{|l|}{ Systemic treatment } \\
\hline Griseofulvin tablet & $52(68.4 \%)$ \\
\hline $\begin{array}{l}\text { Griseofulvin tablet and } \\
\text { cetirizine tablet }\end{array}$ & $4(5.3 \%)$ \\
\hline $\begin{array}{l}\text { Griseofulvin tablet and } \\
\text { loratadine tablet }\end{array}$ & $1(1.3 \%)$ \\
\hline $\begin{array}{l}\text { Griseofulvin tablet and vitamin } \\
\text { B complex tablet }\end{array}$ & $2(2.6 \%)$ \\
\hline $\begin{array}{l}\text { Griseofulvin tablet and } \\
\text { chlorpheniramine tablet } 4 \mathrm{mg}\end{array}$ & $1(1.3 \%)$ \\
\hline Combination treatment & $5(6.6 \%)$ \\
\hline No systemic treatment & $6(7.9 \%)$ \\
\hline No data & $5(6.6 \%)$ \\
\hline \multicolumn{2}{|l|}{ Topical treatment } \\
\hline Urea $10 \%$ cream & $1(1.3 \%)$ \\
\hline Ketoconazole $2 \%$ cream & $2(2.6 \%)$ \\
\hline Miconazole $2 \%$ cream & $2(2.6 \%)$ \\
\hline $\begin{array}{l}\text { Ketoconazole } 2 \% \text { cream and } \\
\text { hydrocortisone } 1 \% \text { cream }\end{array}$ & $1(2.6 \%)$ \\
\hline Combination treatment & $5(1.3 \%)$ \\
\hline No topical treatment & $60(78.9 \%)$ \\
\hline No data & $5(6.6 \%)$ \\
\hline \multicolumn{2}{|l|}{ Combination treatment } \\
\hline $\begin{array}{l}\text { Griseofulvin tablet and urea } \\
10 \% \text { cream }\end{array}$ & $4(5.3 \%)$ \\
\hline $\begin{array}{l}\text { Griseofulvin tablet and } \\
\text { ketoconazole } 2 \% \text { cream }\end{array}$ & $1(1.3 \%)$ \\
\hline No combination treatment & $66(86.8 \%)$ \\
\hline No data & $5(6.6 \%)$ \\
\hline
\end{tabular}

tablet and ketoconazole $2 \%$ cream, and 3 patients $(8.6 \%)$ received a combination of griseofulvin tablet and miconazole $2 \%$ cream. Table 4 shows that the most prescribed systemic drug was griseofulvin tablets, as observed in 52 patients $(68.4 \%) .2$ patients $(2.6 \%)$ received topical therapy of ketoconazole $2 \%$ cream, 2 patients $(2.6 \%)$ received miconazole $2 \%$ cream, and 4 patients $(5.3 \%)$ received a combination therapy of griseofulvin tablet and urea $10 \%$ cream. Table 5 shows that griseofulvin was the most used systemic drug, as observed in 38 patients (71.7\%). The most used topical therapy was urea $10 \%$ cream, miconazole $2 \%$ cream, and ketoconazole $2 \%$ cream, with 2 patients (3.8\%) each. The most used combination therapy was griseofulvin tablet with ketoconazole $2 \%$ cream and griseofulvin tablet with miconazole $2 \%$ cream, which 1 patient (1.9\%) each.

Table 5. The treatment of tinea corporis and tinea cruris

\begin{tabular}{lr}
\hline Treatment & \multicolumn{1}{c}{ Total $(\%)$} \\
\hline Systemic treatment & $38(71.7 \%)$ \\
Griseofulvin tablet & $3(5.7 \%)$ \\
Griseofulvin tablet and cetirizine & \\
tablet & $1(1.9 \%)$ \\
Griseofulvin tablet and & \\
erythromycin tablet & $2(3.8 \%)$ \\
Ketoconazole tablet & $1(1.9 \%)$ \\
Natrium diclofenac tablet, & \\
thiamine tablet, and griseofulvin & \\
tablet & \\
No systemic treatment & $4(7.5 \%)$ \\
No data & $4(7.5 \%)$
\end{tabular}

Topical treatment

Urea cream $10 \%$

$2(3,8 \%)$

Ketokonasol cream 2\%

$2(3,8 \%)$

Mikonasol cream 2\%

$2(3,8 \%)$

No topical treatment

$43(81,1 \%)$

No data

$4(7,5 \%)$

Combination treatment

Griseofulvin tablet and

$1(1,9 \%)$

ketokonazole cream $2 \%$

Griseofulvin tablet and

$1(1,9 \%)$

mikonazole 2 cream \%

$47(88,7 \%)$

No combination treatment

$4(7,5 \%)$ 


\section{DISCUSSION}

In this retrospective study, we found 164 medical records of tinea corporis and cruris patients, and 76 of them were tinea cruris cases. This is similar to studies on superficial fungal infections which results showed that the incidence of tinea corporis and cruris was higher in females than males. ${ }^{6}$ Other studies reported that tinea cruris cases were usually 3 times riskier and had a higher prevalence in males than females. ${ }^{7}$

This may be because males wear occlusive clothing more, and they are more physically active. ${ }^{8}$ However, this does not generalize the entire world population due to the uneven sex composition of the sample. In addition, the number of visits by female patients was higher than that of males. It was possibly because women tend to pay attention to their appearance and aesthetics, further motivating them to seek treatment at the hospital.

We found that the infection of tinea corporis and cruris was higher in youngest-old patients, this result is consistent with previous research conducted by Dyatiara, which was caused by decreased body defense factors with increasing age in the age group and the presence of comorbidities that cause fungal infections. Research reported that the incidence rate of tinea cruris and/or corporis most often occurs in the third- and fourth-decade age group of life as much as $46.2 \%{ }^{8,9}$ As we get older, the body metabolism is slower, and it can cause weight gain, skin creases, more sweat production. Such a condition should be responded to with better self-hygiene and wearing clothes with sweat adsorbing materials. However, the medical records did not include any data on obesity and body mass index (BMI).

We found that fewer pediatric patients were infected with tinea corporis and cruris. Adults suffer from tinea cruris more often than children. This relates to personal hygiene and frequent exposure to sources of transmission such as contact with other sufferers. ${ }^{9}$ Children, however, generally have their activities in a safe and clean environment, and they have regular bathing schedule. Therefore, lowering the tinea corporis and cruris infection in paediatric patients. The data showed that most tinea corporis and cruris cases often occur in private employees. They have packed daily activities which cause sweating, and without better personal hygiene, they are at risk of getting dermatophytosis. ${ }^{10}$ In comparison to other occupations, civil servants, students, or housewives often have their activities indoor. Hot climatic conditions, excessive sweating, tight clothing are the triggering factors for tinea corporis and cruris infections, and such infections can be found in all populations regardless of age and occupation.
We found that itchiness was the most common complaint in patients with tinea corporis and cruris. It was due to sweating. In some cases, the patients also experienced pain caused by scratching, which led to a secondary infection.

Most tinea corporis cases were found in the face and body area, and most tinea cruris cases were found in the groin area. This is in accordance with a journal article. ${ }^{11}$ Non-sweat adsorbing materials would create a hot and humid environment, which is an ideal environment for T. rubrum to grow.

The most common clinical features of patients with tinea corporis, tinea cruris, and tinea corporis and cruris were erythematous macules, firm borders, active margins, scales, and central healing. Dermatophyte infection generally presents a clinical picture in the form of macules and erythematous plaques or hyperpigmentation, clearly demarcated, and papules, vesicles, or pustules can be found at the edges of the skin lesions and accompanied by squama. ${ }^{12}$ The infected area means skin area that forms erythematous macules. The severity of inflammation varies according to illness duration, species of the fungus, and the triggering factors.

We found that most of the tinea corporis and cruris patients sought treatment less than 1 month after the onset. The duration of the most common infections in tinea cruris and tinea corporis et cruris was 1 month, while the duration of tinea corporis infection was two months. ${ }^{11}$ The longer illness duration on tinea corporis patients was possibly caused by patients' attempts for self-medication first. This shows that the majority of patients cared about their health because they immediately sought treatment after symptoms appeared and various clinical features changed in their bodies.

We found that most patients did not have a family history of tinea corporis and cruris. This further strengthens that the triggering factors were habit and climatic conditions, underlying the emergence of tinea corporis and cruris. ${ }^{12}$

There are two criteria used to determine the improvement of the patient's condition, based on clinical examination and laboratory examination. Most of the patients diagnosed with tinea corporis and cruris had positive results $(80.5 \%)$ in $10-20 \% \mathrm{KOH}$ examination. The Wood's lamp examination has never been carried out because this examination was not used to diagnose tinea corporis and cruris patients, but to rule out a differential diagnosis. For example, it is difficult to differentiate between tinea cruris and erythrasma, when the Wood's lamp results positive and showed red brick color, it means erythrasma. In tinea corporis, the Wood's lamp examination was performed 
to rule out the differential diagnosis between tinea corporis and pityriasis versicolor. ${ }^{13}$ Sabouraud Dextrose Agar (SDA) is the most commonly used isolation medium for dermatophytes, and it serves as the medium that forms the basis for most of the morphological descriptions of fungi. The most important examination was $\mathrm{KOH}$ because it is cheap and fast in confirming dermatophytosis. ${ }^{2}$ On those bases, the SDA culture examination was used to identify fungal species is only carried out for certain cases, such as patients with extensive lesions, patients with immunocompromised conditions, or lesions incompatible with tinea corporis or tinea cruris but positive $\mathrm{KOH}$ results. ${ }^{14}$

Griseofulvin was the main therapy of choice in cases of tinea corporis and cruris, and it was the most frequently administered drug in patients with extensive lesions or cases where topical therapy resulted in no improvement. In addition, miconazole $2 \%$ cream and ketoconazole $2 \%$ cream were the most used topical therapy. This was in accordance with the PERDOSKI Clinical Practice Guide. The indication for topical therapy is that if the lesion is not extensive and light, and the indication for systemic therapy is that if the lesion is extensive or more inflamed, often recurs and does not heal with adequate topical drugs or recalcitrant forms. ${ }^{12,15}$

From the dermatophytosis research in the Mycology Division of Dermatology and Venereology Outpatient Clinic Dr. Soetomo General Academic Hospital Surabaya conducted an overview of new cases of dermatophytosis in the Mycology Division of Dermatology and Venereology Outpatient Clinic Dr. Soetomo General Academic Hospital Surabaya in the previous year tended to decline. The therapy that is often used in dermatophytosis is oral/systemic griseofulvin which in general is in accordance with the Clinical Practice Guidelines that apply in Mycology Division of Dermatology and Venereology Outpatient Clinic Dr. Soetomo General Academic Hospital Surabaya, with a fairly good recovery rate. Filling in data using medical records is quite complete by filling in complete patient data when taking anamnesis on the category of identity, main complaint, length of illness, family history, trigger factors, location of lesions, supporting laboratories, and therapy will make diagnosis easier, facilitate patient treatment, and complete data collection.

\section{REFERENCES}

1. Verma S, Verma G, Sharma V, Bhagra S, Negi A, Tegta GR. Current Spectrum of Dermatophytosis in a Tertiary Care Hospital of North India - A 6-Year
Clinico-Mycological Study. J Med Sci Clin Res 2017; 05(03): 19488-94.

2. Craddock LN, Schieke SM. Superficial Fungal Infection. In: Kang S, Amagai M, Bruckner AL, Enk AH, Margolis DJ, McMichael AJ, et al., editors. Fitzpatrick's Dermatology. 9th Edition. New York: McGraw-Hill Education; 2019. p. 2925-51.

3. Hayette M, Sacheli R. Dermatophytosis, Trends in Epidemiology and Diagnostic Approach. Curr Fungal Infect Rep 2015;9(3):164-79.

4. Mahajan R, Sahoo A. Management of tinea corporis, tinea cruris, and tinea pedis: A comprehensive review. Indian Dermatol Online J 2016; 7(2): 77-86.

5. Leung A, Lam J, Leong K, Hon K. Tinea corporis: an updated review. Drugs Context 2020; 9: 1-12.

6. Oninla OA, Oninla SO. Superficial Mycoses in Relation to Age and Gender. Br J Med Med Res 2016; 13(5): 1-10.

7. Oktaviana N, Kawilarang AP, Damayanti. Patient Profile Of Tinea Corporis In Dr. Soetomo General Hospital, Surabaya From 2014 To 2015. Jurnal Berkala Epidemiologi 2018; 6(3): 200-8.

8. Madhavi S, Rama Mao M, Jyothsna K. Mycological study of Dermatophytosis in rural population. Ann Biol Res. 2011; 2(3): 88-93.

9. Devy D, Ervianti E. Studi Retrospektif: Karakteristik Dermatofitosis. Berkala Ilmu Kesehatan Kulit dan Kelamin 2018; 30(1): 66-72.

10. Sondakh CE, Pandaleke TA, Mawu FO. Profil dermatofitosis di Poliklinik Kulit dan Kelamin RSUP Prof. Dr. RD Kandou Manado periode Januari-Desember. e-CliniC 2016; 4(1): 1-7.

11. Yuwita W, Ramali LM, Miliawati R. Karakteristik Tinea Kruris dan/atau Tinea Korporis di RSUD Ciamis Jawa Barat. Berkala Ilmu Kesehatan Kulit dan Kelamin 2016; 28(2): 42-51.

12. Siswati AS, Ervianti E. Dermatologi Infeksi. In: Bramono K, Suyoso S, Indriatmi W, Ramali L, Widaty S, Ervianti E, editors. Dermatomikosis Superfisialis. 2nd ed. Jakarta: Badan Penerbit FK UI; 2013. p. 58-69.

13. Fuller LC, Barton RC, Mohd Mustapa MF, Proudfoot LE, Punjabi SP, Higgins EM. British Association of Dermatologists' guidelines for the management of tinea capitis 2014. Br J Dermatol 2014; 171(3): 454-63.

14. Poluri LV, Indugula JP, Kondapaneni SL. Clinicomycological Study of Dermatophytosis in South India. J Lab Physicians 2015; 7(2): 84-9.

15. Yossela T. Diagnosis and Treatment of Tinea cruris. J MAJORITY. 2015; 4(2): 122-8. 